\title{
Immunocompatibility of Rad-PC-Rad liposomes in vitro, based on human complement activation and cytokine release
}

Sofiya Matviykiv ${ }^{\mathrm{a}}$, Marzia Buscema ${ }^{\mathrm{a}}$, Gabriela Gerganova ${ }^{\mathrm{a}}$, Tamás Mészáros ${ }^{\mathrm{b}, \mathrm{c}}$, Gergely Tibor Kozma $^{\mathrm{b}, \mathrm{c}}$, Ute Mettal ${ }^{\mathrm{d}}$, Frederik Neuhaus ${ }^{\mathrm{d}}$, Takashi Ishikawa ${ }^{\mathrm{e}}$, János Szebeni ${ }^{\mathrm{b}, \mathrm{f}, \mathrm{f}}$, Andreas Zumbuehl ${ }^{\mathrm{d}}$, and Bert Müller ${ }^{\mathrm{a} *}$

${ }^{a}$ Biomaterials Science Center, Department of Biomedical Engineering, University of Basel, Allschwil, Switzerland

${ }^{b}$ Nanomedicine Research and Education Center, Institute of Pathophysiology, Semmelweis University, Budapest, Hungary

${ }^{c}$ SeroScience Ltd., Budapest, Hungary

${ }^{d}$ Department of Chemistry, University of Fribourg, Fribourg, Switzerland

ePaul Scherrer Institute, Villigen, Switzerland

${ }^{f}$ Department of Nanobiotechnology and Regenerative Medicine, Miskolc University, Miskolc, Hungary

Submitted: March 26, 2018;

Accepted: April 19, 2018

Published: April 25, 2018

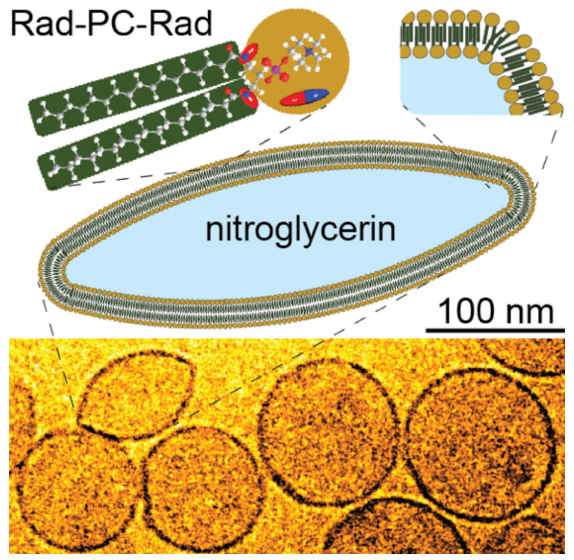

Graphical Abstract: The mechanoresponsive Rad-PC-Rad liposomes, designed to deliver a vasodilator drug to stenosis, are stable even at elevated body temperatures. The question is whether these nano-containers with a specific shape present adverse effects similar to liposomal drugs in vitro or they don't.

\section{Abstract}

Liposomal drug delivery systems can protect pharmaceutical substances and control their release. Systemic administration of liposomes, however, often activate the innate immune system, resulting in hypersensitivity reactions. These pseudo-allergic reactions can be interpreted as activating the complement system. Complement activation destroys and eliminates foreign substances, either directly through opsonization and the formation of the membrane attack complex (MAC), or by activating leukocytes and initiating inflammatory responses via mediators, such as cytokines. In this study, we investigated the in vitro immune toxicity of the recently synthesized Rad-PC-Rad liposomes, analyzing the liposome-induced complement activation. In five human sera, Rad-PC-Rad liposomes did not induce activation, but in one serum high sensitivity via alternative pathway was detected. Such a behavior in adverse phenomena is characteristic for patient-to-patient variation and, thus, the number of donors should be in the order of hundreds rather than tens, hence the present study based on six donors is preliminary. In order to further prove the suitability of mechano-responsive Rad-PC-Rad liposomes for clinical trials, the production of pro-inflammatory cytokines was examined by human white blood cells. The concentrations of the pro-inflammatory cytokines, IL-6, IL-12p70, TNF- $\alpha$, and IL-1 $\beta$, induced by Rad-PC-Rad liposomal formulations, incubated with whole blood samples, were smaller or comparable to PBS (negative control). Because of this favorable in vitro hemocompatibility, in vivo investigations using these mechano-responsive liposomes should be designed. ${ }^{i}$

\footnotetext{
* Corresponding author: phone +41 61207 5430, bert.mueller@unibas.ch, Biomaterials Science Center, Department of Biomedical Engineering, University of Basel, Gewerbestrasse 14, 4123 Allschwil, Switzerland
} 


\section{Keywords}

Nanomedicines, non-spherical liposomes, immune toxicity, complement activation, hypersensitivity reactions, pro-inflammatory cytokines

\section{Purpose and Rationale}

The artificial diamidophospholipid Rad-PCRad has been synthesized recently [1]. Rad-PCRad liposomes aim to preferentially deliver the vasodilator molecules to the stenosed parts of blood vessels. Liposomes, administered intravenously, are immediately exposed to a complex environment of blood cells and proteins. The adsorption of plasma proteins on the surface of liposomes may not only decrease the therapeutic efficiency and biodistribution, but also may result in immunotoxicity. The toxicities which represent the most common safety issues and reasons for nanomedicines failure include complement-mediated reactions and cytokine-mediated inflammation, which can result in anaphylaxis. Therefore, in this study we investigated complement activation and release of pro-inflammatory cytokines, mediated by Rad-PC-Rad liposomes. The immunotoxicity can be also influenced by the therapeutic payload or addition of surface ligands. Therefore, the comparison between nitroglycerin-loaded and drug-free liposomes, as well as PEGylated and non-PEGylated liposomes was evaluated. The physicochemical properties of nanomedicines are crucial to determine their interaction with the immune system. Hence, we characterized the size, zeta potential, and phospholipid concentration of the Rad-PC-Rad liposomes.

\section{Introduction}

The latest progress in the nanomedicine field has resulted in the development of smart nanocontainers for drug delivery applications, including liposomes. Liposomes can improve delivery, targeting, and therapeutic efficacy of the drug and, at the same time, increase the halflife of the drug, lower its effective dose, and reduce toxic side effects $[2,3]$. Previously, our research team reported on shear stress sensitive Pad-PC-Pad liposomes for targeted delivery of a vasodilator to constricted arteries $[4,5]$. A further in vivo investigation of Pad-PC-Pad phospholipids was limited owing to their phase transition temperature at $37^{\circ} \mathrm{C}$. Very recently, we have reported on a more thermally stable phospholipid formulation, such as Rad-PC-Rad
[1]. This lipid exhibits a bilayer main phase transition temperature of $44.7^{\circ} \mathrm{C}$ and preserves the responsiveness for mechanical triggers [1].

The immediate treatment of arterial occlusion generally involves intravenous injection of nitroglycerin (NTG), which acts as a vasodilator. Systemic administration of NTG may cause severe adverse effects including hypotension and diminished blood perfusion to the heart. The targeted delivery of NTG via the incorporation into shear stress sensitive liposomes may reduce these side effects. The direct contact of liposomes with blood carries the risk of immediate activation of the innate immune system [6]. This may result not only in the reduction of the drug' $\mathrm{s}$ efficacy, but also in the appearance of hypersensitivity reactions (HSRs) [6-8]. The main function of the immune system is to protect the organism from invading pathogens. It can, however, also develop an immune response against non-pathogenic objects, such as nanometer-size liposomes. Therein, the recruitment of the complement system is an important step in the recognition and elimination of foreign materials. The complement system is a group of approximately 30 plasma- and membranebound proteins [9]. Their protective function leads to the release of active components, which cause opsonization, inflammation, and the generation of the membrane attack complex (MAC) [10]. According to the current literature, the complement activation occurs via the three established routes: classical, lectin, and alternative pathways [10]. One can discriminate between these pathways by identifying the presence of unique protein fragments: $\mathrm{C} 4 \mathrm{~d}$ (classical and lectin pathways) and $\mathrm{Bb}$ (alternative pathway) [11]. Activation of either pathway results in the turnover of the $\mathrm{C} 3$ protein, which is followed by the production of the anaphylatoxins $\mathrm{C} 3 \mathrm{a}$ and $\mathrm{C} 5 \mathrm{a}$, and the formation of the MAC (C5b-9). The release of anaphylatoxins causes leukocyte chemotaxis and the production of pro-inflammatory cytokines, which finally induce inflammation (Figure 1). The excessive production of anaphylatoxins can be harmful and may cause anaphylactic shock or even organ failure at 
relevant concentrations [12]. Binding of the proteins to the liposomes depends also on their composition, size, geometry, surface charge, and hydrophobicity that can act as immunological adjuvant and trigger strong immune response $[6,13]$. The undesirable activation of the complement system can be caused by systemically administered liposomes, such as Doxil ${ }^{\circledR}$ (PEGylated liposomal doxorubicin) and AmBisome ${ }^{\circledR}$ (liposomal amphotericin B), leading to the development of HSRs, termed complement activation-related pseudoallergy (CARPA) [7, 8]. Approximately $2-10 \%$ of patients may adversely react to intravenously administered liposomal formulations with mild-to-severe hypersensitivity reactions [8]. CARPA develops at the first exposure and its symptoms involve almost all organ systems [14]. Some of the most important safety concerns for nanoparticle failure are related to the toxicities caused by complement activation-mediated reactions and cytokine-mediated inflammation [15]. Therefore, it is recommended that liposomes intended for intravenous injection are tested in vitro and in vivo for the potential activation of complement system, as a preclinical immune toxicity test [16]. The assessment of the liposomal physicochemical properties and their impact on complement activation is also an important objective in the development of nanometer-size therapeutics.

The production of pro-inflammatory cytokines in vitro is considered a marker of cytokineassociated immunotoxicity in vivo [15] and screening for these toxicities early in preclinical characterization will help to avoid potentially toxic candidates in nanomedicine development. Recently, Wolf-Grosse et al. reported about cytokine secretion in a complement-dependent manner [17]. They state that cytokine response was generally mostly due to C5a activation, as it is the most potent pro-inflammatory mediator released upon $\mathrm{C}$ activation [17]. Therefore, in order to prevent the potential immunotoxicity in vivo, we studied the effect of Rad-PC-Rad liposomes on the production of complement proteins and pro-inflammatory cytokines.

\section{Experimental design}

In the present article, we address the possibilities that Rad-PC-Rad liposomes, loaded with NTG solution, would activate the complement system and stimulate the release of pro-inflammatory cytokines, thus raising concern about potential risk for CARPA or cytokine storm. Thus, we have measured in vitro complement activation in human sera and the release of the pro-inflammatory cytokines, in human whole blood and isolated leukocytes, upon incubation with Rad-PC-Rad liposomes. The complement pathway activation products $\mathrm{C} 4 \mathrm{~d}$ and $\mathrm{Bb}$, and terminal complement complex SC5b-9 were measured using an enzyme linked immunosorbent assay (ELISA), and the release of the pro-inflammatory cytokines IL-1 $\beta$, IL-6, IL-8, IL-12, TNF- a was measured using a cytometric bead array test. In addition, we analyzed the liposomal physicochemical properties, in terms of liposomes size and zeta potential, using dynamic light scattering (DLS), and estimated membrane thickness from the micrographs, obtained by cryogenic transmission electron microscopy (cryo-TEM).

\section{Materials and Methods}

\section{Materials}

1,3-Diheptadecanamidopropan-2-yl [trimethylammonio]ethyl) phosphate (Rad-PCRad) was synthesized and purified according to the recently reported protocol [1]. Figure 2 shows the structural formula of the Rad-PCRad phospholipid. Table S1 lists all the materials used for the experiments.

Human sera from six healthy volunteers and whole blood samples from two healthy donors were obtained through an institutionally approved phlebotomy protocol at Semmelweis University (Budapest, Hungary). Human sera were stored at a temperature of $-80^{\circ} \mathrm{C}$ until usage. Whole blood samples were freshly collected into sterile hirudin-treated tubes and immediately employed for experiment. Freshly drawn blood, used for leukocytes isolation, was provided by the Hungarian National Blood Transfusion Service. 


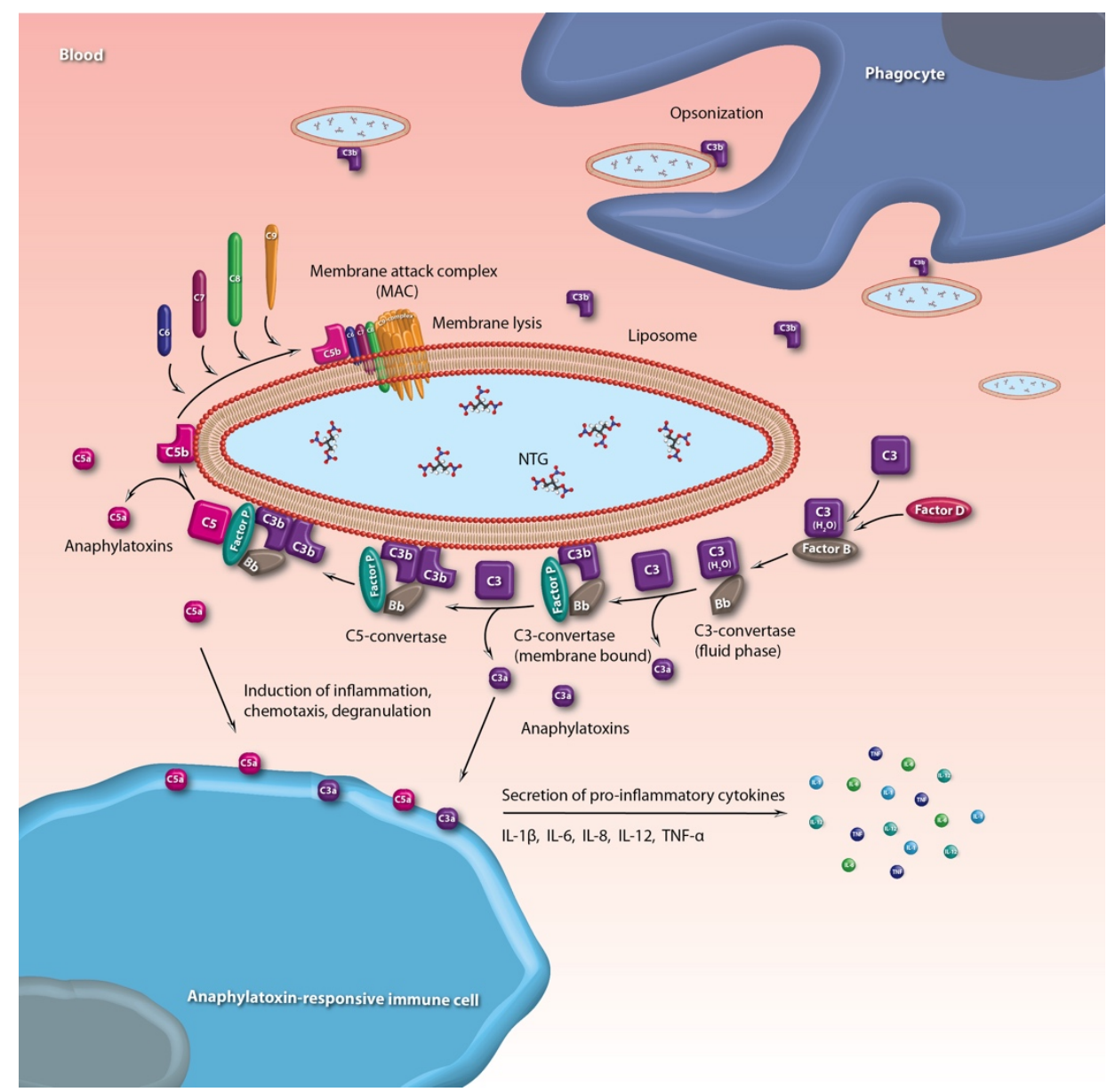

Figure 1. Schematic representation of complement (C) activation triggering pro-inflammatory cytokines due to anaphylatoxin binding to anaphylatoxin-receptor positive cells (e.g., mast cells, basophils, neutrophils, platelets and pulmonary intravascular macrophages). In the case of Rad-PC-Rad liposomes, C activation proceeds through the alternative pathway. On this pathway C3 directly binds to liposomal phospholipid head-groups. Factor B binds to the newly attached C3b, and again becomes susceptible to cleavage by factor D. Membrane-bound C3bBb is unstable until it is bound by properdin protein (factor P). Stabilized C3-convertase rapidly generates large amounts of C3b that bind more factor B, resulting in dramatic amplification of C3b. Membrane-bound C3b serves as an opsonin and a binding tag for phagocytic cells. Addition of C3b to C3-convertase results in the formation of C5-convertase, which cleaves C5 into C5b, and proceeds to form the MAC. Cleavage of $C 5$ also results in the formation of C5a anaphylatoxin. Together with $C 3 a$, the C5a fragment binds to the surface C receptors of mentioned allergy mediating cells. C3a and C5a receptors, after binding small anaphylatoxins, mediate the allergic reaction by stimulating the release of vasoactive mediators (e.g., histamine, thromboxanes, leukotrienes, etc.).

\section{Liposome preparation}

Four Rad-PC-Rad/DSPE-PEG 2000 phospholipid formulations were prepared, namely $\mathrm{R} 1$, R2, R3, and R4 (see Table 1). Lipids were dissolved in chloroform in molar ratios as listed in Table 1. The preparation of the liposomal formulations is described in detail in ref. [18]. The samples were purified through sterile filters and stored at a temperature of $4{ }^{\circ} \mathrm{C}$ until usage.

\section{Characterization of liposomal formulations}

Phospholipid concentration. A colorimetric assay (phosphate test 2.0) [19] was used for the determination of the phospholipid content of the liposomal formulations after extrusion and purification. Here, the phosphate moiety in the head group of the phospholipids was a measure of the total phospholipid concentration.

Table 1. Composition of Rad-PC-Rad liposomal formulations

\begin{tabular}{lccc}
\hline \multirow{2}{*}{ Label } & \multicolumn{2}{c}{$\begin{array}{c}\text { Lipid composition } \\
\text { (molar \%) }\end{array}$} & $\begin{array}{c}\text { Loading } \\
\text { buffer }\end{array}$ \\
\cline { 2 - 3 } & $\begin{array}{c}\text { Rad-PC- } \\
\text { Rad }\end{array}$ & $\begin{array}{c}\text { DSPE- } \\
\text { PEG }_{2000}\end{array}$ & \\
\hline R1 & 100 & - & saline \\
R2 & 95 & 5 & saline \\
R3 & 100 & - & NTG \\
R4 & 95 & 5 & NTG \\
\hline
\end{tabular}


To exclude the variations in lipid concentration, the concentration of each liposomal formulation (R1, R2, R3, and R4) was diluted with $0.9 \%$ sodium chloride solution (saline) to a total lipid concentration of $10 \mathrm{mg} / \mathrm{mL}$. In addition, a set of the diluted formulations (R1d, $\mathrm{R} 2 \mathrm{~d}$, R3d, and R4d) with $5 \mathrm{mg} / \mathrm{mL}$ of phospholipids were prepared for the in vitro immunoassays to examine the impact of the lipid concentration on the complement activation level (see Figures S2 and S3).

Physicochemical characteristics. The liposome average diameter, polydispersity index (PDI) and zeta $(\zeta)$ potential were obtained by DLS performed at a temperature of $25^{\circ} \mathrm{C}$ using a DelsaMax PRO (Beckman Coulter, USA). The suspensions were diluted 100 times in saline prior to the measurements.

Liposome morphology. The morphology of four Rad-PC-Rad formulations was studied using cryogenic transmission electron microscopy (cryo-TEM) (JEM2200FS, JEOL, Tokyo, Japan). The samples, diluted with saline in the ratio $1: 1$, were imaged as previously reported [1, $18]$.

Encapsulation efficiency. The encapsulation efficiency of Rad-PC-Rad liposomes for passive loading with nitroglycerin was determined indirectly by measuring the peak of a glucose-trifluoroacetic acid adduct by electrospray ionization mass spectrometry (ESI-MS) on a Bruker esquire HCT ion trap mass spectrometer (Bruker Corporation, USA) [18].

Liposomal release. Two Rad-PC-Rad liposomal formulations, i.e. with and without DSPE-PEG, were loaded with 5(6)carboxyfluorescein (CF) buffer, and prepared as described in ref. [1]. Seven aliquots with a volume of $2 \mathrm{~mL}$ were separated into $5 \mathrm{~mL}$ glass vials and kept for selected periods of time $(0,5$, $10,20,40 \mathrm{~min})$ at a temperature of $37^{\circ} \mathrm{C}$. The $\mathrm{CF}$ release was quantified using a fluorospectrometer (SpectraMax 2, Bucher Biotec AG, Switzerland) with the wavelengths of $485 \mathrm{~nm}$ for excitation and $538 \mathrm{~nm}$ for emission. Sample fluorescence at a temperature of $20{ }^{\circ} \mathrm{C}$ served as a negative control $\left(F_{0}\right)$. As a positive control for the maximum dye release $\left(F_{100}\right)$, liposomal samples were heated to a temperature of $65^{\circ} \mathrm{C}$, above the lipids transition temperature of $44.7^{\circ} \mathrm{C}$. The release fraction at the selected time point $x$ was calculated according to:

$$
\text { Release }(\%)=\frac{F_{x}-F_{0}}{F_{100}-F_{0}}
$$

where $F_{x}$ is the fluorescence at time $x$.

\section{Complement immunoassay}

Activation of human sera with liposomes. Human sera from six healthy donors were thawed and kept at a temperature of $4^{\circ} \mathrm{C}$ during the experiment. Due to the limited amount of serum available, the sera \#5 and \#6 were prepared as pools from distinctive donors in the ratios 1.5:1 and 7.8:1, respectively. The liposomal suspensions in the two concentrations were added to the sera of each donor in the ratio of $1: 3$. Saline and nitroglycerin were used as negative controls. FDA-approved liposomal drugs, with recorded cardio-toxicity effects and activation of the complement system in sensitive patients, Doxil $^{\circledR}(2 \mathrm{mg} / \mathrm{mL}$ doxorubicin, $12.77 \mathrm{mg} / \mathrm{mL}$ phospholipids, used as provided) and AmBisome $^{\circledR}(17.975 \mathrm{mg} / \mathrm{mL}$ amphotericin B, $4.02 \mathrm{mg} / \mathrm{mL}$ phospholipids, reconstituted with injection water) were employed as well [7]. Zymosan $(1.2 \mathrm{mg} / \mathrm{mL})$, known as activator of the complement system, was used as positive control. Each activation mixture was incubated at a temperature of $37^{\circ} \mathrm{C}$. The concentration of the terminal complement complex SC5b-9 was investigated over time. The incubation was terminated after $5,10,20$, and 40 minutes by adding $10 \mathrm{mM}$ EDTA.

\section{ELISA immunoassays}

The ELISA assays were carried out following the manufacturer' $\mathrm{s}$ protocol. The optical density was measured with a 96-well plate reader (FLUOstar Omega, BMG Labtech, Germany) at a wavelength of $450 \mathrm{~nm}$ for SC5b9, $\mathrm{Bb}, \mathrm{C} 3 \mathrm{a}$ and $\mathrm{C} 5 \mathrm{a}$ as well as at a wavelength of $405 \mathrm{~nm}$ for $\mathrm{C} 4 \mathrm{~d}$.

\section{Cytokine immunoassay}

Isolation of leukocytes from buffy coat. A volume of $400 \mathrm{~mL}$ of buffy coat (BC), a pool of white blood cells (WBCs) concentrates of four healthy volunteers, were obtained from the Hungarian National Blood Transfusion Service within 24 hours of blood withdrawal. Altogether three BC pools were used, each consisting of four donors. Leukocytes were further concentrated two times by mixing with DPBS (w/o $\mathrm{CaCl}_{2}, \mathrm{MgCl}_{2}$ ) in 1:1 ratio and 
centrifuged for a period of ten minutes at a velocity of $750 \mathrm{G}$ and a temperature of $4^{\circ} \mathrm{C}$. To lyse the remaining erythrocytes, distilled water at a temperature of $4^{\circ} \mathrm{C}$ was added to the BC (4:1 ratio) for 20 seconds. Lysis was stopped by adding one volume hyperosmotic salt solution (containing $1.8 \%$ of $\mathrm{NaCl}$ ). After washing with ice-cold DPBS (w/o $\mathrm{CaCl}_{2}$, $\mathrm{MgCl}_{2}$ ) for platelets elimination, WBCs were re-suspended in $\mathrm{R} 5$ medium.

Qualitative and quantitative analysis of isolated leukocytes. The concentration of WBCs, in three independent blood packages was determined. Viable cells were detected using FITC Annexin V apoptosis detection kit, see Table S4. The staining procedure was performed according to the manufacturer' $\mathrm{s}$ instructions. Leukocytes were further diluted or concentrated to reach the necessary concentration of $\sim 10^{8}$ cells $/ \mathrm{mL}$. The cell viability was also checked after cell isolation and treatments by test materials and control agents. The viability of cells before and after treatments were always higher than 98\%, except for the positive control, Table S5.

Activation of $B C$ leukocytes with liposomes. Freshly isolated leukocytes from three independent blood packages were separately incubated with four Rad-PC-Rad liposomal formulations in the ratio 7:1. Samples, with a concentration of $4 \mathrm{mg} / \mathrm{mL}$, were incubated for four hours at a temperature of $37^{\circ} \mathrm{C}$ on a shaker plate. The incubation was stopped by EDTA (final concentration $10 \mathrm{mM}$ ). Cell culture supernatants were further used for mixing with cytokine capture beads. The assay was performed according to the suggested protocol the manufacturer provided with the kit.

Activation of human whole blood with liposomes. Freshly collected whole human blood from two donors was separately incubated with four Rad-PC-Rad liposomal formulations at a concentration of $4 \mathrm{mg} / \mathrm{mL}$, following the same procedure as described in the section above. The distinctive step was the incubation time. Here samples were incubated for a period of six hours. Whole blood samples had no R5 medium, instead they contained their own plasma.

Qualitative and quantitative analysis of leukocytes originated from whole blood samples. An aliquot of human blood from two donors was stained as described in the section above. Cell viability was determined before and after treatments by test materials and control agents. The percentage of viable cells was more than $97 \%$, except for the positive control, Table S5.

Cytometric bead array test. The human inflammatory cytokines kit was used to quantitatively measure interleukin- $1 \beta$ (IL- $\beta$ ), interleukin-6 (IL-6), interleukin-8 (IL-8), interleukin-12p70 (IL-12p70), tumor necrosis factor a (TNF- $\alpha$ ), and interleukin-10 (IL-10) protein levels in the studied samples. The assay was carried out following the manufacturer' $\mathrm{s}$ instructions. The beads fluorescence was recorded by flow cytometry using a FACScan instrument (BD Biosciences, USA), and the data were analyzed using the Kaluza Analysis 1.5 software (Beckman Coulter, USA).

\section{Statistical analysis}

Statistical analysis was carried out using GraphPad Prism 6 (GraphPad Software Inc., USA). Data from the ELISA samples (Figures 4 and 7), except zymosan, were compared with saline as negative control after 40 minutes of incubation. Significance of differences between the groups was determined by non-parametric Kruskal-Wallis test, followed by Dunn's multiple comparisons test. $P$-values lower than 0.05 were considered as statistically significant.

\section{Results}

\section{Characterization of Rad-PC-Rad liposomal}

\section{formulations}

The lipid concentration of Rad-PC-Rad ranged from 10 to $20 \mathrm{mg} / \mathrm{mL}$, and the mean diameter of the liposomes in the suspensions was around $100 \mathrm{~nm}$ and varied from 95 to $140 \mathrm{~nm}$ (see Table 2). Measurements after 20 days showed that the PEGylated liposomes did not change their size, whereas the non-PEGylated ones displayed an increase from 140 to $270 \mathrm{~nm}$ (R1) and from 115 to $200 \mathrm{~nm}$ (R3).

Table 2 lists the measured zeta potential values of the Rad-PC-Rad formulations. Pure Rad-PCRad samples revealed positive $\zeta$ potential values, between +1.3 (R1) to $+4.7 \mathrm{mV}$ (R3), while PEGylated samples turned to negative potentials, from -2.0 (R4) to $-4.5 \mathrm{mV}(\mathrm{R} 2)$.

The size and morphology of Rad-PC-Rad liposomes, evaluated using cryo-TEM imaging, is represented in Figure 2. These micrographs show intact spherical, lenticular, and faceted 
unilamellar liposomes below their main phase transition temperature. The percentage of faceted liposomes within samples was $46 \%$ (R1), 72\% (R2), 42\% (R3), and 52\% (R4).

Table 2. Characteristics of Rad-PC-Rad liposomal formulations.

\begin{tabular}{|c|c|c|c|c|c|c|}
\hline Label & $\begin{array}{l}\text { Lipid } \\
\text { composition }\end{array}$ & $\begin{array}{l}\text { Lipid } \\
\text { content } \\
(\mathrm{mg} / \mathrm{mL})\end{array}$ & $\begin{array}{l}\text { Mean } \\
\text { diameter } \\
(\mathrm{nm})^{*}\end{array}$ & PDI* & $\begin{array}{l}\text { Membrane } \\
\text { thickness } \\
(\mathrm{nm})\end{array}$ & $\begin{array}{c}\zeta \text { potential } \\
(\mathrm{mV})^{*}\end{array}$ \\
\hline R1 & $\begin{array}{l}\text { Rad-PC-Rad } \\
\text { (saline) }\end{array}$ & $10.17 \pm 0.02$ & $138.6 \pm 3.5$ & $0.15 \pm 0.03$ & $3.27 \pm 0.14$ & $+1.29 \pm 0.37$ \\
\hline $\mathrm{R} 2$ & $\begin{array}{l}\text { Rad-PC- } \\
\text { Rad/DSPE- } \\
\text { PEG }_{2000} \text { (saline) }\end{array}$ & $18.39 \pm 0.13$ & $106.5 \pm 1.9$ & $0.14 \pm 0.02$ & $3.60 \pm 0.21$ & $\begin{array}{c}- \\
4.52 \pm 1.05\end{array}$ \\
\hline $\mathrm{R} 3$ & $\begin{array}{l}\text { Rad-PC-Rad } \\
\text { (NTG) }\end{array}$ & $13.62 \pm 0.72$ & $114.5 \pm 0.4$ & $0.06 \pm 0.03$ & $3.27 \pm 0.19$ & $+4.65 \pm 0.42$ \\
\hline R4 & $\begin{array}{l}\text { Rad-PC- } \\
\text { Rad/DSPE- } \\
\text { PEG }_{2000} \text { (NTG) }\end{array}$ & $21.75 \pm 3.35$ & $97.0 \pm 0.6$ & $0.12 \pm 0.01$ & $3.50 \pm 0.24$ & $\begin{array}{c}- \\
2.08 \pm 0.51\end{array}$ \\
\hline
\end{tabular}

* Data were recorded immediately after sample preparation.

The addition of DSPE-PEG (see Figure 2D and $2 \mathrm{~F}$ ), led to the co-existence of flat circular disks and unilamellar liposomes. Depending on the disk orientation, they appear either as small rods with high contrast (red-colored arrows), or, when seen from the top, as circular structures with low uniform contrast (Figure 2D, right).

The liposome membrane thickness was estimated from the cryo-TEM projections of the appropriately oriented membranes (Figure 2). We have measured the individual thicknesses of 100 membranes and found the mean values of $\mathrm{R} 1$ to be $(3.27 \pm 0.14) \mathrm{nm}$ and of $\mathrm{R} 3$ to be (3.27 \pm 0.19$) \mathrm{nm}$, which indicates the interdigitation of the Rad-PC-Rad leaflets. Interdigitation may be one of the driving forces in the formation of faceted liposomes [1]. Samples loaded with DSPE-PEG, i.e. R2 and $\mathrm{R} 4$, tended to result in higher mean values. The values correspond to $(3.60 \pm 0.21) \mathrm{nm}$ and $(3.50 \pm 0.24) \mathrm{nm}$, respectively. The mean diameters of the liposomes, derived from cryoTEM images, were $10-15 \%$ smaller than those obtained from DLS data.

The encapsulation efficiency of NTG-loaded samples was estimated from the ESI-MS measurements. The calculation is based on the $100 \%$ ESI-MS signal of pure NTG and liposome size. The employed NTG solution contained glucose as an excipient, therefore, the NTG encapsulation was determined indirectly. The integral of the glucose-trifluoroacetic acid adduct was evaluated after NTG incorporation. The ratio between these values determines the percentage of NTG encapsulation efficiency (see Table S2). The values correspond to $38 \%$ (R3) and $12 \%(\mathrm{R} 4)$.

In order to measure the membrane permeability, a release test of CF-loaded liposomes at a temperature of $37^{\circ} \mathrm{C}$ was performed under static conditions (Figure 3). Both, Rad-PC-Rad and Rad-PC-Rad/DSPE-PEG samples demonstrated an immediate spontaneous release of $17 \%$ and $8 \% \mathrm{CF}$, respectively. During another 40 minutes of incubation the release level increased to $36 \%$ (Rad-PC-Rad) and 33\% (Rad-PC-Rad/DSPE-PEG). The initial $\mathrm{CF}$ release trend line of both samples was distinctive, however after 25 minutes the spontaneous release level of the two formulations was comparable and reached about $35 \%$. 
A<smiles>CCCCCCCCCCCCCCCCC(=O)NCC(CNC(=O)CCCCCCCCCCCCCC)OP(=O)([O-])OCC[N+](C)(C)C</smiles>

B

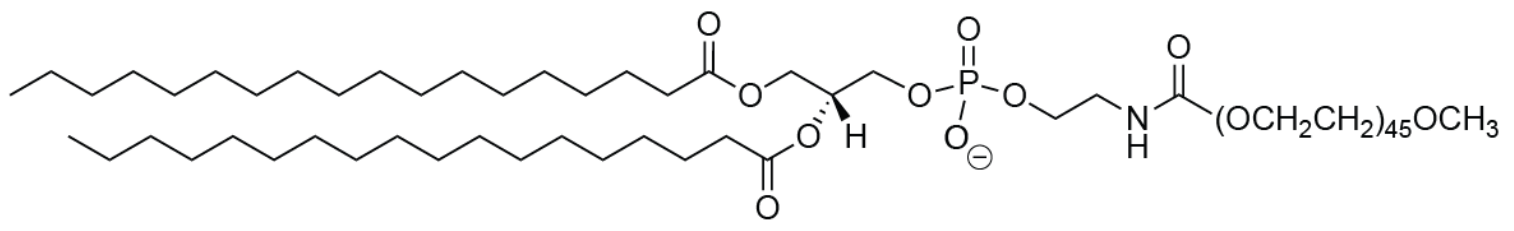

C

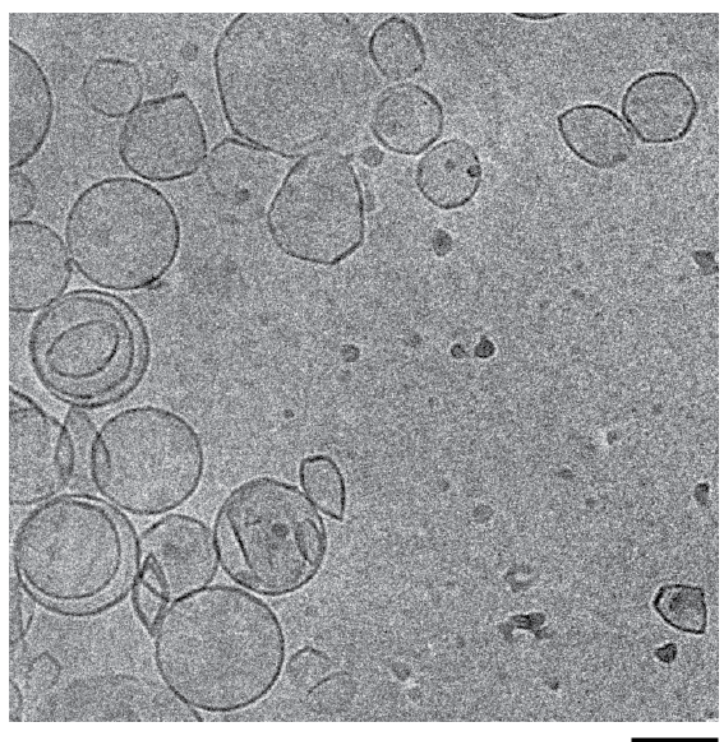

$\mathbf{E}$

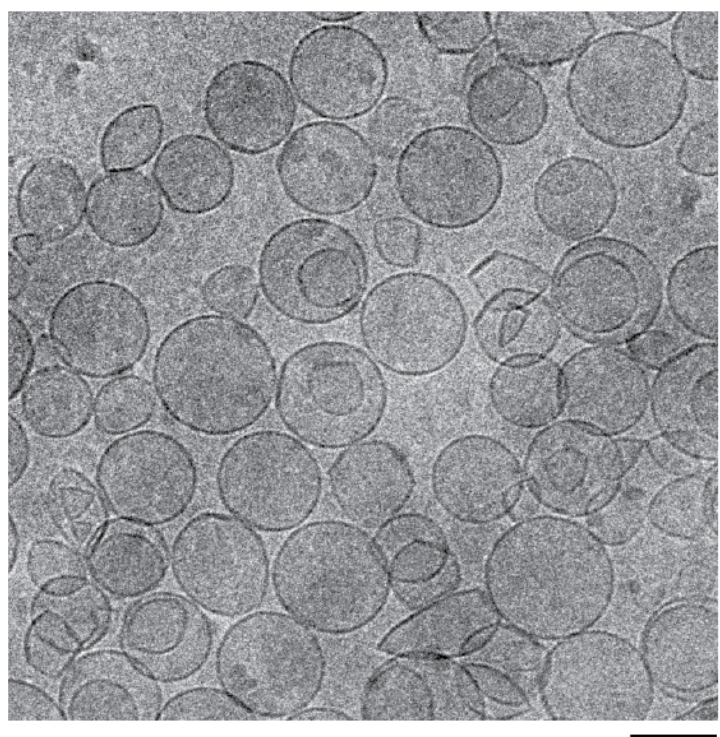

D

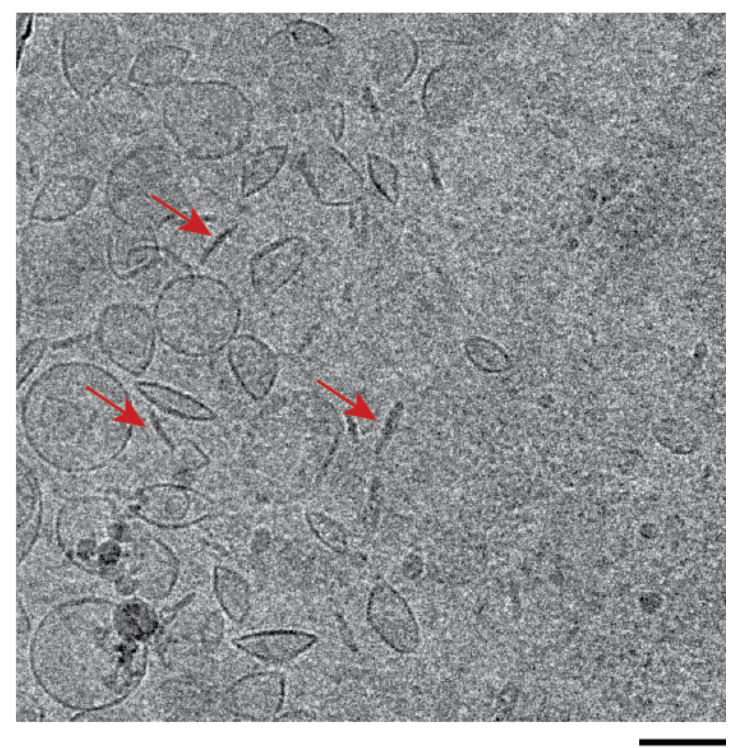

$\mathbf{F}$

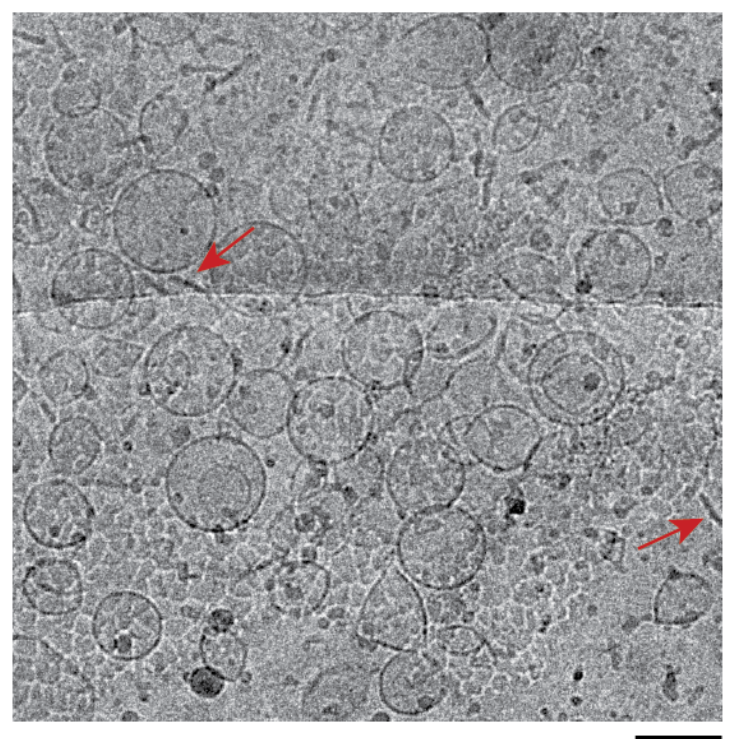

Figure 2. The structure of the two phospholipids used for the liposome preparation: Rad-PC-Rad (A) and DSPE-PEG (B). Cryo-TEM micrographs of the liposomal formulations: $R 1$ (C), $R 2$ (D), R3 (E), R4 (F). Scale bars are $100 \mathrm{~nm}$. The samples contain spherical and faceted liposomes. The incorporation of DSPE-PEG caused the formation of bicelles indicated by the red-colored arrows. 


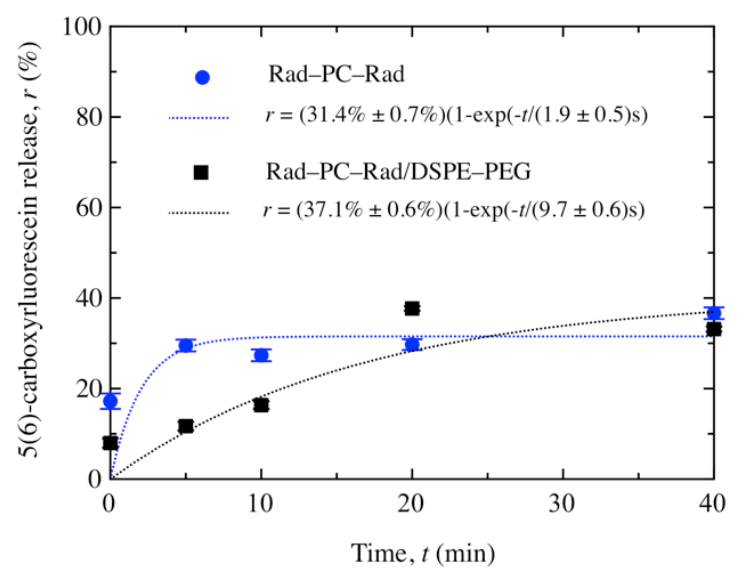

Figure 3. The release of 5(6)-carboxyfluorescein containing liposomes formulated from Rad-PC-Rad and Rad-PC-Rad/DSPE-PEG. The samples were heated to a temperature of $37^{\circ} \mathrm{C}$ and kept at constant temperature for a period of 0, 5, 10, 20, and 40 minutes. The final spontaneous $\mathrm{CF}$ release was about $35 \%$. The data are represented as mean values, error bars indicate standard deviation $(n=5)$.

\section{Complement immunoassays}

Figure 4A displays the level of SC5b-9 (TCC), which is a marker for the activation of the complement system. The mean values of the six donors demonstrate that the liposomal formulations R1 and R3 have caused an elevenand 15-fold increase in SC5b-9 concentration in comparison to the negative control (saline), whereas R2 and R4 have raised the SC5b-9 concentration by a factor of eight or nine, respectively. Noticeably, Donor \#5 (gray hexagonal) showed a tremendous increase of TCC in the four Rad-PC-Rad liposomal formulations, which largely contributed to the increased mean values of each liposomal sample. The increase of SC $5 \mathrm{~b}-9$ was at the same level with its positive control, namely about 28fold compared with saline (Table S3). Certainly, the contribution of Donor \#5 into the mean value of all six donors, caused a deviation of mean values up to four times. Doxil ${ }^{\circledR}$ and AmBisome $^{\circledR}$ samples did not reveal a substantial difference in the level of SC5b-9 between the six donors. Doxil ${ }^{\circledR}$ samples showed less than two-fold increase, while AmBisome ${ }^{\circledR}$ demonstrated more than nine-fold elevation of the TCC protein compared to saline. Zymosan caused a considerably high level of complement activation, namely 55-fold, and was excluded from the diagrams to present the differences between the tested samples in a clearer way. Our findings were statistically confirmed. NTG, which was chosen as another negative control, was compared to saline and no statistical difference was identified. R1 and R3 samples showed very significant to extremely significant differences compared to the negative control. In contrast, the samples R2 and R4 did not reveal a statistically significant difference versus saline. The SC5b-9 level caused by Doxil ${ }^{\circledR}$ among the six sera, showed no statistical difference compared to saline. Certainly, AmBisome ${ }^{\circledR}$ was detected to be extremely significant towards the negative control.

Figure 4B demonstrates the level of C4d protein, which is an experimental marker for the activation of the classical and the lectin pathways. Neither the Rad-PC-Rad liposomes, nor the FDA-approved liposomal formulations revealed a significant increase of $\mathrm{C} 4 \mathrm{~d}$ protein concentration. The mean values show a less than four-fold increase in comparison to the negative control.

Figure $4 \mathrm{C}$ represents the level of $\mathrm{Bb}$ fragment as an experimental marker for alternative complement system activation. The serum from Donor \#5 towards Rad-PC-Rad liposomal formulation showed four to eight-fold elevation of $\mathrm{Bb}$ concentration, whereas the other five donors demonstrated less than three-fold increase compared to saline. All six sera were similarly sensitive to the AmBisome ${ }^{\mathbb{B}}$ with an increase of four times above the negative control, revealing a strong statistical significance. A significant serum reactivity towards Doxil ${ }^{\circledR}$ was not observed.

The detection of C3a (Figure 4D) revealed no significant difference between the highly sensitive donor and the others. Most of the values were within the error bars. Rad-PC-Rad liposomes and Doxil ${ }^{\circledR}$ showed values between two- and three-times higher in comparison to saline, while AmBisome ${ }^{\circledR}$ caused an elevation of C3a similar to that one of the positive control - a three- to four-fold increase. 
A

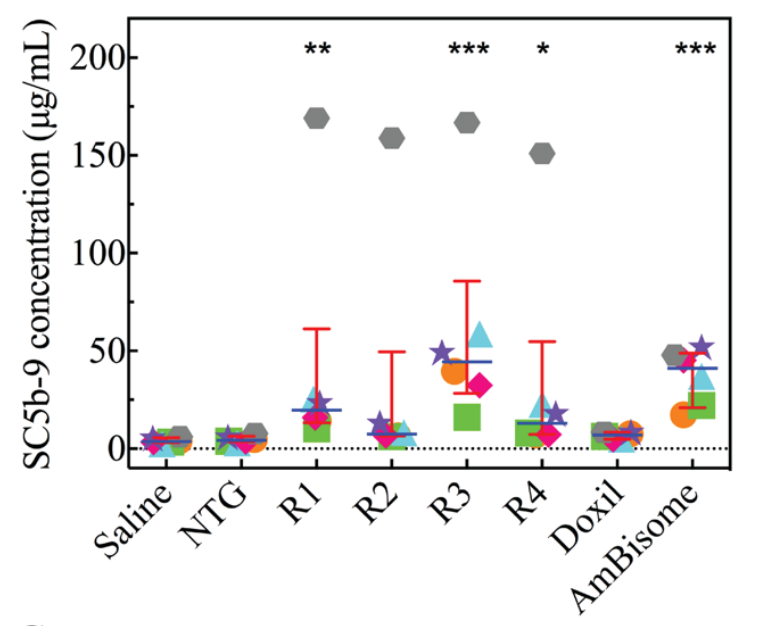

C

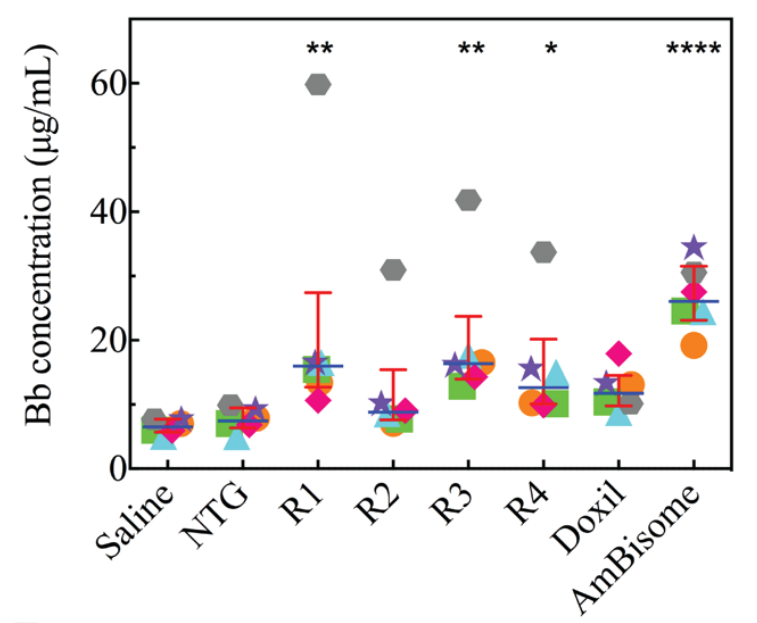

E

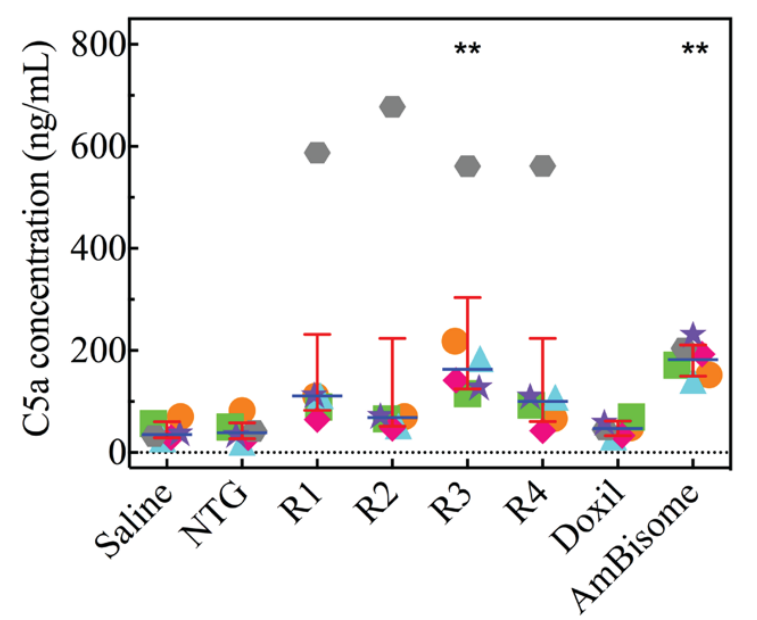

B

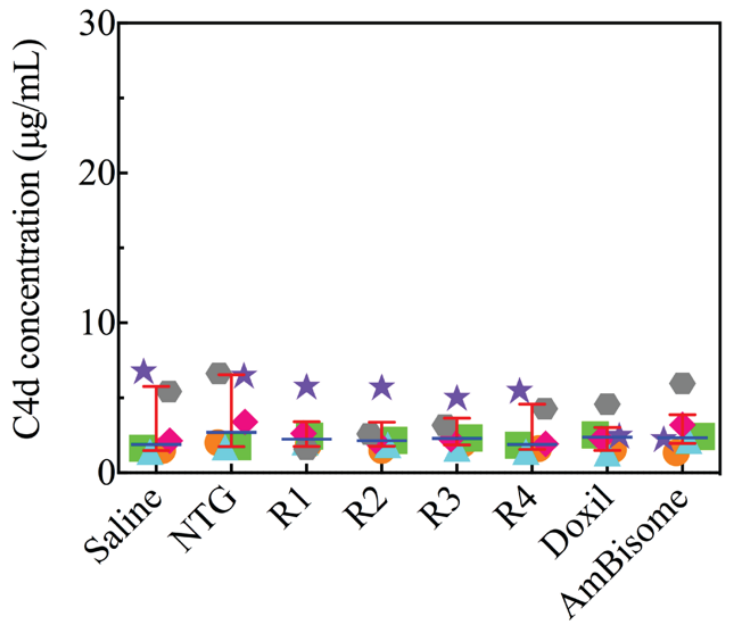

D

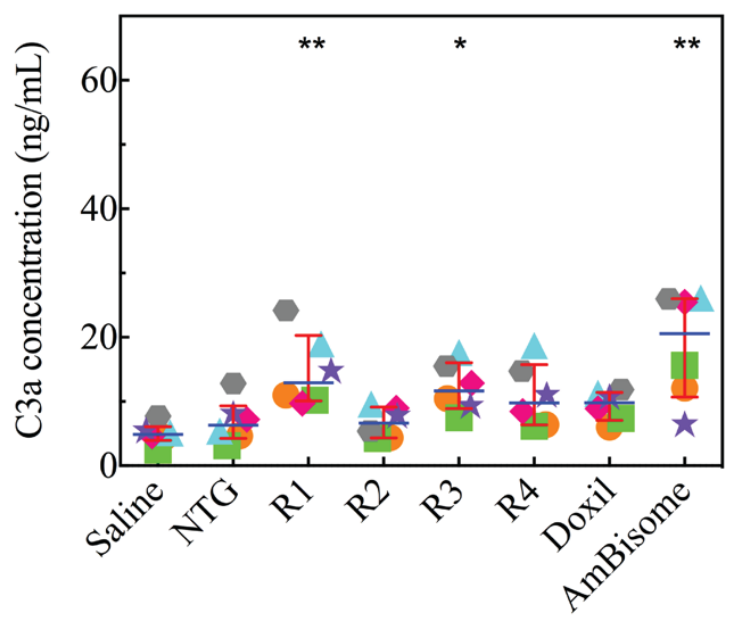

Donor \#1

Donor \#2

Donor \#3

Donor \#4

Donor \#5

Donor \#6

Figure 4. The concentration of SC5b-9 (A), C4d(B), Bb (C), C3a (D), and C5a (E) complement proteins. Human sera from six independent donors were incubated for a period of 40 minutes at a temperature of $37^{\circ} C$ with saline, nitroglycerin (NTG), Rad-PC-Rad liposomal suspension of selected composition (R1, R2, R3, R4), Doxil ${ }^{\circledR}$ and AmBisome ${ }^{\circledR}$. Saline solution was chosen as negative control. NTG was used as another negative control. Non-PEGylated liposomal formulations caused a higher level of $C$ activation, mainly via the alternative pathway. Highly sensitive serum towards artificial Rad-PC-Rad liposomes was identified. This activation of the $C$ cascade resulted in the increased production of C5a anaphylatoxin. The positive control zymosan caused substantially higher levels of complement activation. The data are represented as median, including error bars derived from the interquartile range among six donors. Each symbol and color represents data from a single donor. Significance of differences among the groups was determined by non-parametric Kruskal-Wallis test, followed by Dunn's multiple comparisons test. P-values lower than 0.05 were considered as statistically significant. 
A

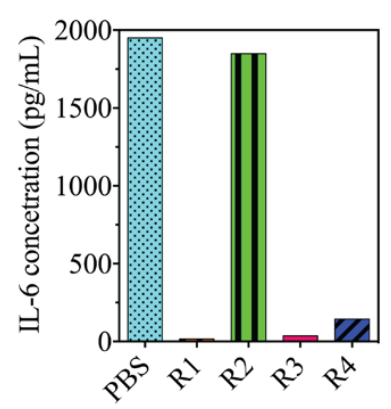

C

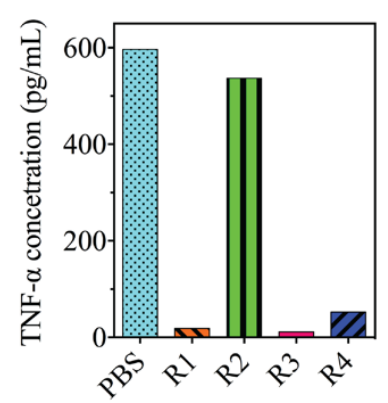

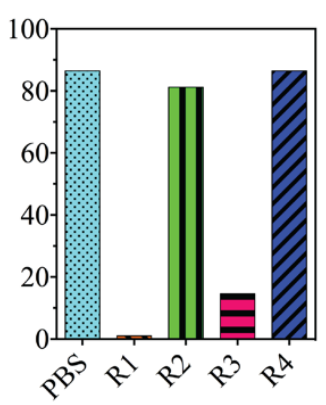

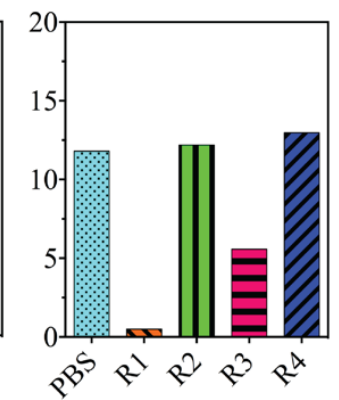

B
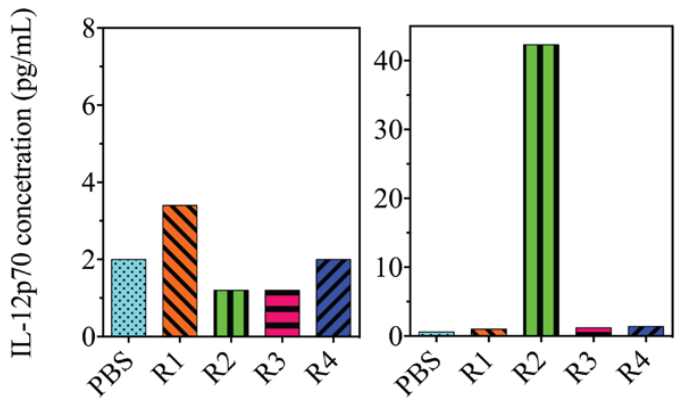

D

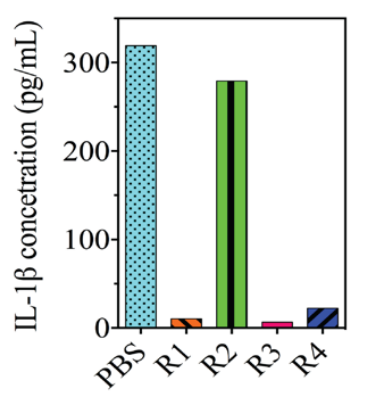

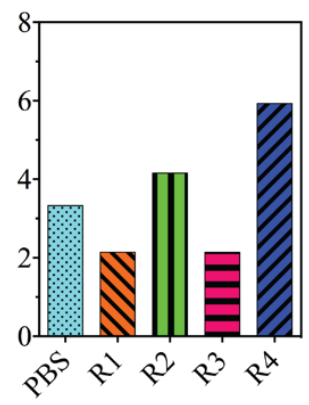

Figure 5. Concentration of pro-inflammatory cytokines IL-6 (A), IL-12p70 (B), TNF- a (C), and IL-1 $\beta$ (D) induced by RadPC-Rad liposomal formulations (R1-R4) incubated with whole blood samples from two donors (first donor: left panels, second donor: right panels) for a period of six hours at a temperature of $37^{\circ} \mathrm{C}$. PBS was chosen as negative control. The effect of Rad-PC-Rad liposomes upon production of cytokines was lower or comparable to the negative control. Less than two-fold increase in the IL-12p70 concentration was observed by R1 treatment (first blood sample). The second blood sample revealed a highly elevated concentration of IL-12p70 induced by R2. IL-1 $\beta$ concentration of the second donor' $s$ blood was close to the detection limit of the kit. The positive control zymosan caused a considerably high level of complement activation, which was mostly above the top standard concentration and was therefore excluded from the graph. The data represent a single value from each donor.

For the activation of SC5b-9, Bb, and C3a, Donor \#5 demonstrated a similar trend, where the reactivity towards Rad-PC-Rad samples was higher for the R1 and R3 samples compared to R2 and R4.

Donor \#5 showed a significant increase in C5a concentration in comparison to the other five donors (Figure 4E). The values were elevated 17- to 21-fold above the negative control, and the trend was distinctive from the one previously observed (SC5b-9, $\mathrm{Bb}$ and $\mathrm{C} 3 \mathrm{a}$ ). Here, sample R2 revealed the highest C5a level, whereas R1, R3 and R4 were similar. The increase of the C5a concentration detected by the other five donors was below four times the baseline for the Rad-PC-Rad samples and Doxil $^{\circledR}$, while AmBisome ${ }^{\circledR}$ displayed a fourfold increase

\section{Cytokine immunoassay}

The concentration of the inflammatory cytokines is listed in Table S6: IL-6, IL-12p70, TNF- $\alpha$, IL-1 $\beta$, IL-10 and IL-8. Figures 5 and 6 represent the levels of the identified proinflammatory cytokines in whole blood and BC cells, respectively, caused by Rad-PC-Rad liposomal formulations.

The concentration of each cytokine was compared to saline, which served as a negative control. Whole blood samples were collected from two donors and revealed a substantial difference in the sensitivity towards the studied samples. The effect of Rad-PC-Rad liposomal formulations on the production of proinflammatory cytokines were lower or comparable to the negative control. Less than a two-fold increase in the cytokine concentration was caused by R1 and R4 samples, in case of IL-12p70 (first blood sample) and IL-1 $\beta$ (second blood sample), respectively, see diagrams in Figure 5B and 5D. The concentration of IL-1 $\beta$ (second blood sample) was below the detection limit of the kit (see Table S6). Surprisingly, the second donor showed a large increase in production of IL$12 \mathrm{p} 70$ in response to the R2 sample, even higher than the positive control. However, as no additional donor samples were available, we can't conclude the importance of this increase. 
A

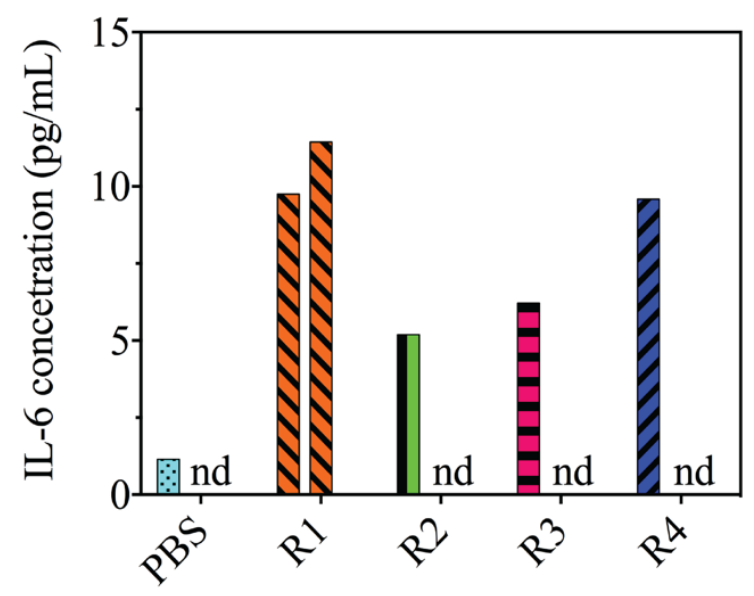

C

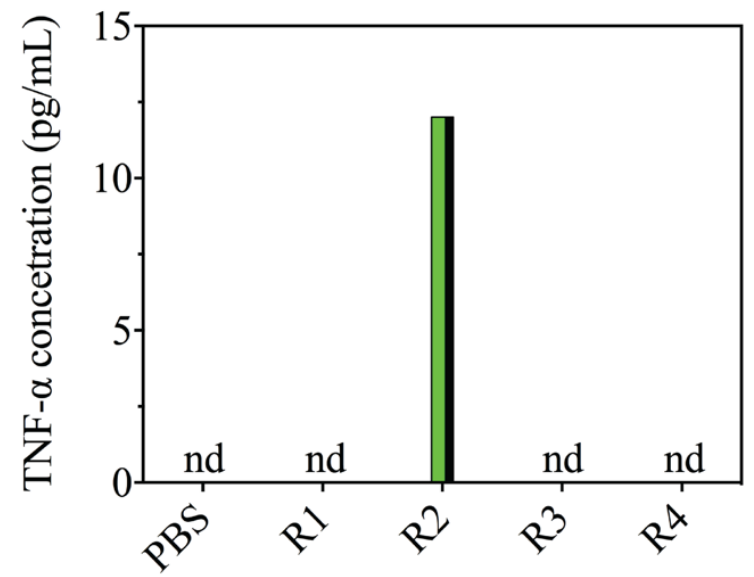

B

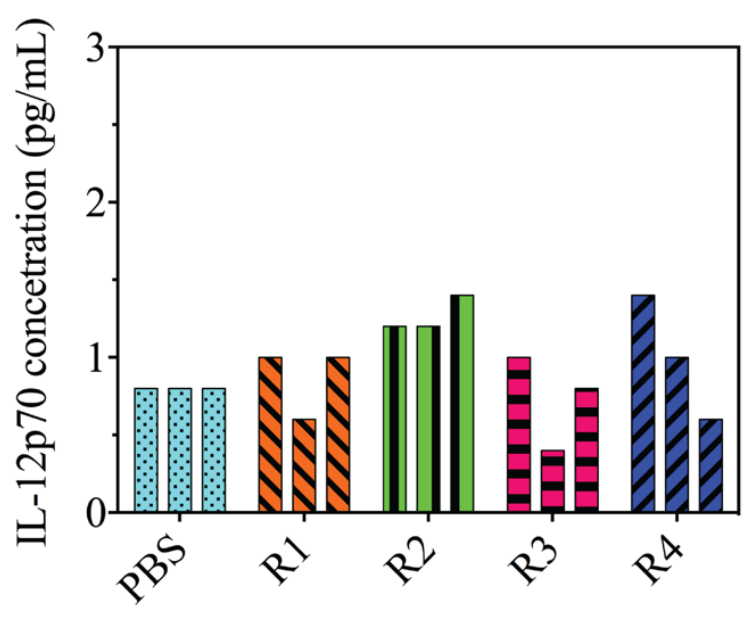

D

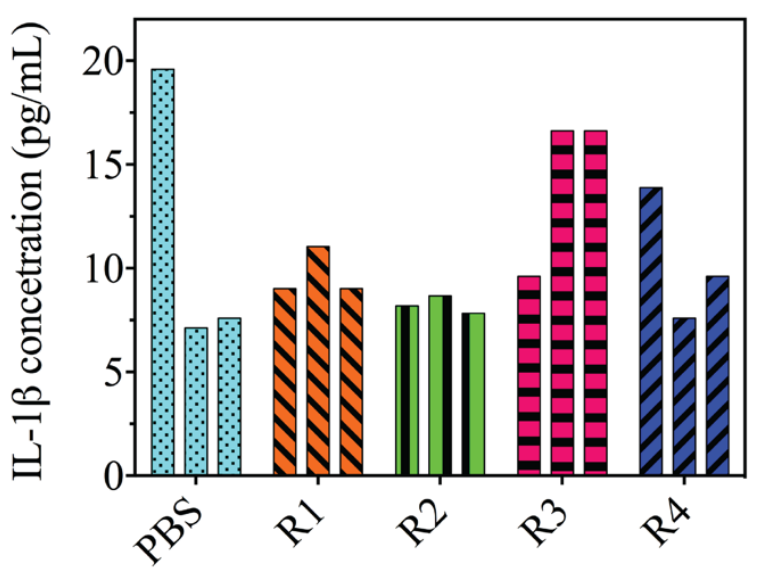

Figure 6. Concentration of pro-inflammatory cytokines induced by Rad-PC-Rad liposomal formulations incubated with isolated leukocytes from three buffy coat pools, each consisting of four donors. Buffy coats were incubated with R1-R4 for a period of four hours at a temperature of $37^{\circ}$ C. PBS was chosen as negative control. The Rad-PC-Rad formulations caused an elevated level of IL-6 in a part of the buffy coat samples. Insubstantial elevation of IL-12p70 was identified in R2 treated group in each BC. The increase in the level of TNF- a was observed only in one of the buffy coats by R2 sample. The other Rad-PC-Rad liposomal formulations were comparable with PBS. The positive control zymosan caused a considerably higher level of complement activation, which was above the top standard concentration and was therefore excluded from the graph. The data represent a single value from each buffy coat. The label "nd" means not detected.

The concentration of IL-10 was detected only in the samples treated with the positive control. The level of IL- 8 gave false positive results and was above the top standard concentration, see Table S6. Therefore, it was excluded from the study.

Rad-PC-Rad formulations caused an elevated level of IL- 6 , by four- to nine-fold, in one of three BCs compared to PBS (see Figure 6A). The concentration of IL- 6 caused by R5 medium, however, was comparable to the one caused by R1 and R4 (see Table S6). Another $\mathrm{BC}$ sample demonstrated an increased level of IL-6, caused only by the R1 sample. A raise in the IL- 6 concentration caused by the third tested BC sample was not detected by any of the liposomal formulations (see Figure 6A). In case of IL-12p70, incubation with four Rad-PC-Rad formulations resulted in less than two-fold increase of the cytokine level (see Figure 6B). Moreover, in some of the cases, IL-12p70 levels stimulated by Rad-PC-Rad liposomes were lower than those of the negative control. A surprising increase in the concentration of TNF- a was observed in one of the BC samples by R2 (Figure 6C). None of the other samples elicited an increase in the TNF- a cytokine. In case of IL-1 $\beta$, only R3 demonstrated a two-fold increase of the cytokine above the negative control (see Figure 6D) whereas the other Rad- 
PC-Rad liposomes revealed an increase of IL$1 \beta$ of less than a factor of two. In summary, the cytokines levels caused by Rad-PC-Rad liposomal formulations were comparable with those caused by the negative control.

\section{Discussion}

The objective of the study was to investigate the immune response towards the recently developed artificial Rad-PC-Rad liposomes loaded with NTG. For this purpose, we measured the level of $\mathrm{C}$ activation markers and pro-inflammatory cytokines in vitro. We performed the physicochemical characterization of Rad-PC-Rad liposomes and discussed their potential influence upon the activation of the immune system.

\section{Characterization of Rad-PC-Rad liposomal formulations}

The surface charge of Rad-PC-Rad liposomal suspensions was characterized using the $\zeta$ potential, which is one of the principal indicators of the colloid stability. A potential of $\pm 30 \mathrm{mV}$ has been considered as the limit, above which a colloidal system becomes stable [20]. Rad-PC-Rad liposomes are non-charged nanometer-sized species with relatively low repulsive forces. They tend to aggregate. To improve the stability of the Rad-PC-Rad liposomes, we incorporated DSPE-PEG molecules into the liposomes, which cause a steric repulsion between lipid bilayers [21]. Furthermore, it resulted in the alteration of the $\zeta$-potential to negative values. This alteration is a consequence of the negatively charged DSPE-PEG molecules owing to its phosphodiester moiety [22].

The morphology of Rad-PC-Rad liposomes can deviate from the spherical shape. The liposomes often exhibit irregular facets (Figure $2)$. The co-existing spherical and facetted liposomes are two forms that may differ in the molecular fraction of the lipid components. Therefore, we have performed differential scanning calorimetry and found that upon repeated heating and cooling additional peaks arose (see Figure S1). This observation clearly indicates that various phases have become coexistent. The occurrence of faceted liposomes is explained by the formation of an intermolecular hydrogen-bonding network at the hydrophobic-hydrophilic interface of the lipids and membrane interdigitation, as recently studied for Rad-PC-Rad in detail [1].
The PEG-containing liposomes, R2 and R4, sometimes appear as flat circular disks (see micrographs $D$ and $F$ in Figure 2). In fact, these features originated from tilted bicelles or micelles [23, 24]. It should be noted that DSPEPEG is known as a micelle-forming species [25, 26]. The spontaneous formation of bicelles is related to the phase separation between RadPC-Rad and DSPE-PEG. The formation of bicelles without an internal cavity led to the decrease of NTG encapsulation by $26 \%$. This shortcoming has to be addressed in future investigations by balancing the PEGincorporation to optimize the liposomes, stability and minimize bicelles formation.

The cobblestone-like features in the micrograph D of Figure 2 may originate from PEGylated lipids, which failed to form liposomes. The samples have to be frozen for the cryo-TEM data acquisition. The higher viscosity of PEGylated samples results in the formation of thick ice, which impedes the imaging, see micrographs D and F of Figure 2.

\section{Complement immunoassays}

We examined the biological effects of Rad-PC$\mathrm{Rad}$ liposomes on $\mathrm{C}$ activation. $\mathrm{C}$ activation by liposomes depends on several physicochemical factors, including lipid composition, liposome size, morphology, and surface charge [27-29]. Larger liposomes are more prone to activate the complement system than smaller liposomes [30]. This observation explains the increased level of $\mathrm{C}$ proteins by the R1 and R3 samples, which diameters were larger and increased with time owing to aggregation. The importance of geometric factors on the assembly of complement convertases [30] or provoking cardiopulmonary distress in pigs [31] can also be a pivotal parameter in combating adverse reactions. The impact of geometry and topology on complement activation was identified by Moghimi et al. and Wibroe et al. [28, 31]. The cryo-TEM images represented in Figure 2 show Rad-PC-Rad liposomes with faceted and spherical shapes as well as disk-like PEGylated phospholipid-based bicelles. Therefore, the study on adverse reactions of rod-and disk-like particles reported recently [31] is of particular value. Even though this study reports on Cindependent adverse injection reactions, it clearly demonstrates the impact of the particle morphology on the immune cells recognition.

The incorporation of PEG into liposomes is 
considered to improve $\mathrm{C}$ compatibility and prolong liposomes circulation [32]. Their infusion, however, can give rise to HSRs [7, 33, 34]. PEGylated liposomes are able to trigger $\mathrm{C}$ activation due to the presence of the anionic phosphate-oxygen moiety of the PEGylated phospholipid [35]. Positive or negative surface charge may also enhance $\mathrm{C}$ activation [36], for example by insertion of the negatively charged PEGylated phospholipids. Such negative $\zeta$ potential values were identified in the PEGcontaining Rad-PC-Rad liposomal formulations. The $\mathrm{C}$ activation-promoting activity of negative surface charges on liposomes was already discussed previously [7]. In our study, only minor differences were observed in PEGylated samples versus negative control.

Another interesting phenomenon was observed, when drug molecules were encapsulated into a liposomal cavity (e.g., Doxil ${ }^{\circledR}$, prednisolone). The $\mathrm{C}$ activation was considerably higher than for the liposomes of the same size and composition but without the entrapped drug [32, 37]. In case of Doxil ${ }^{\circledR}$, this phenomenon was explained by changes in the liposomal morphology arising from the drug loading procedures [28]. Here, we observed that NTGcontaining samples (R3 and R4) caused a slightly increased level of $\mathrm{C}$ proteins, in comparison to their drug-free counterparts. NTG alone did not have any significant impact on $\mathrm{C}$ activation $(P<0.05)$.

The release test showed that $17-36 \%$ of loaded buffer was spontaneously released during the incubation. These observations suggest that the drug incorporation into the liposomes causes physicochemical changes to the liposomal formulation. Together with a partial release of the drug it may have a synergistic effect on $\mathrm{C}$ activation. The frequency of HSRs from FDA-approved liposomal drugs varies, for example $\sim 10 \%$ with Doxil $^{\circledR}$ and $\sim 30 \%$ with AmBisome ${ }^{\circledR}$ [7]. AmBisome $^{\circledR}$, a highly negatively charged liposome formulation, was found to be a very strong $\mathrm{C}$ activator [7]. AmBisome ${ }^{\circledR}$ significantly differs from Doxil ${ }^{\circledR}$, which causes strong $\mathrm{C}$ activation only in certain sensitive sera. This feature was confirmed in our experiment. AmBisome $^{\circledR}$ caused the formation of significantly higher $(P<0.05)$ levels of all $\mathrm{C}$ proteins (Figure 4).

The increased level of TCC led us to determine the pathway of $\mathrm{C}$ activation. We found that the classical and lectin pathways were not involved in the $\mathrm{C}$ activation, but could confirm that the alternative pathway was involved, in agreement with previous results on Pad-PC-Pad liposomes [11].

It has to be noted that one of the six human sera showed an anomaly. This pooled serum, \#5, was highly sensitive to the Rad-PC-Rad liposomes. Although the pooling of sera from different donors is common for in vitro studies, it should be handled with care and the ratio seems to play an important role: the volumes from each serum should be equal [38]. As this phenomenon has not been considered in the present study, the experimental data were evaluated excluding Donor \#5 (see Figure 7).

The differences between the sera provide evidence for the utility of in vitro $\mathrm{C}$ assays and mimic the clinical situation. HSRs are mostly minor and transient; however, life-threatening reactions can happen occasionally in a hypersensitive individual. It has been suggested that for those donated sera, which cause $\mathrm{C}$ activation more than four times the baseline level, the related human being has an elevated risk of HSRs development [33]. The mean level of SC5b-9, within four sera (for R2 and R4) was below four times baseline (saline) (see Figure 4A). Therefore, one could expect that these donors do not carry a risk for developing hypersensitivity reactions in vivo. R1 and R3, however, showed a five- and eleven-fold increase in the SC5b-9 concentration, which means that non-PEGylated Rad-PC-Rad liposomes might trigger the development of HSRs in vivo. Our previous studies demonstrate that at high phospholipid concentration PadPC-Pad liposomes induce weak to none complement activation in vitro, but no significant changes in the hemodynamic parameters, nor anaphylactic reactions were observed in vivo [18, 39].

The identification of the reactive Donor \#5 motivated further studies. Those studies showed that inflammatory mediators, such as $\mathrm{C} 3 \mathrm{a}$ and $\mathrm{C} 5 \mathrm{a}$, became activated. The increased level of the anaphylatoxins potentially causes strong pro-inflammatory or anaphylactic responses [6, 10, 40]. Although the $\mathrm{C} 3 \mathrm{a}$ concentration caused by Rad-PC-Rad liposomes was below three-fold the baseline level, the non-PEGylated liposomal samples were elevated significantly $(P<0.05)$. In 
addition, the Rad-PC-Rad liposomes provoked $\mathrm{C}$ activation that resulted in an increased level of $\mathrm{C} 5 \mathrm{a}$ anaphylatoxin. It is known that $\mathrm{C} 3 \mathrm{a}$ and $\mathrm{C} 5 \mathrm{a}$ are major contributors to the release of proinflammatory cytokines $[6,9,40]$. These fragments serve as ligands for receptors on leukocytes that trigger inflammation and release of pro-inflammatory cytokines, including IL-1 $\beta$, TNF- $\alpha$, IL- 6 and the highly potent chemokine IL-8 [9]. The activation of leukocytes with $\mathrm{C} 3 \mathrm{a}$ and $\mathrm{C} 5 \mathrm{a}$ is the most relevant property of these proteins for immune compatibility. C5a induces degranulation, chemoattraction and acts with IL- $1 \beta$ and TNF$\alpha$ to induce an acute immune response [10]. Recently, a direct link between $C$ activation and the secretion of cytokines, caused by iron nanoparticles was presented [17]. Therefore, to prevent any other potential immunotoxicity in vivo, we studied the effect of Rad-PC-Rad liposomes on the production of proinflammatory cytokines.

\section{Cytokine immunoassay}

The identification of a highly reactive donor to Rad-PC-Rad liposomes, as demonstrated by a consistently and significantly higher level of complement proteins and $\mathrm{C} 5 \mathrm{a}$ anaphylatoxin prompted us to investigate the proinflammatory response to Rad-PC-Rad. Measuring the cytokine release allows estimating the inflammatory properties of the tested species. To quantify the impact of RadPC-Rad liposomes on cytokines' production, we used isolated leukocytes and human whole blood. Our results obtained with whole blood showed differences between the two donors. Multiple factors have impact on the cytokine level, such as intra-individual differences in physical activity and exercising. Chronic exercise training results in decreased levels of many circulating cytokines [41]. One of the donors was physically active, which explains the reduced cytokine level. The samples R1 and $\mathrm{R} 2$ were above the negative control owing to the IL-12p70 production. The IL-1 $\beta$ level was below the detection limit. The results indicate that Rad-PC-Rad liposomes hardly cause the production of pro-inflammatory cytokines in whole blood. Liposomes induce cytokine production as a result of the physicochemical parameters, i.e. size below $100 \mathrm{~nm}$, surface charge (cationic lipids), and hydrophobicity [13]. We did not observe cytokine production upon the positive charge. Both positively charged Rad-PC-Rad liposomal formulations $\mathrm{R} 1$ and R3 showed unrelated variations of the elevated cytokine levels. The results show no indication that the liposomal size plays a role in cytokine production. The PEG-free samples R1 and R3 with higher mean liposome diameters do not lead to elevated cytokines' production. In all cases the response to the liposomes showed levels of pro-inflammatory cytokines comparable to the ones of saline or R5 medium (see Table S6). The main causes of increased cytokine production, however, are unknown, as immunotoxicity mechanisms relate to the composition of nanomaterials, the cell type and cycle, the animal model, and the disease status. The biological variability may have more impact on the immune reaction than expected. The possibility that certain results are attributable to endotoxin contamination [42] are valid, even though the samples were passed through a sterilized filter, and experiments were conducted under a sterilized hood.

IL-8 is an essential chemokine involved in the recruitment of neutrophils to the site of inflammation. More than $50 \%$ of the tested nanomaterials, which induce the activation of pro-inflammatory cytokines, caused exclusive production of IL-8, without inducing TNF- a and IL- $1 \beta$. Besides, more than $50 \%$ of such inducers were liposomes and emulsions [15]. This phenomenon is not fully understood; however, a study suggests the involvement of oxidative stress [43].

Within the present study, the cytometric bead array test gave false positive results of IL-8. This means that the concentration of IL-8 in the negative and positive controls was above the standard concentration of the kit (see Table S6). Thus, we cannot conclude whether Rad-PCRad liposomes induce the production of IL- 8 or not. 
A

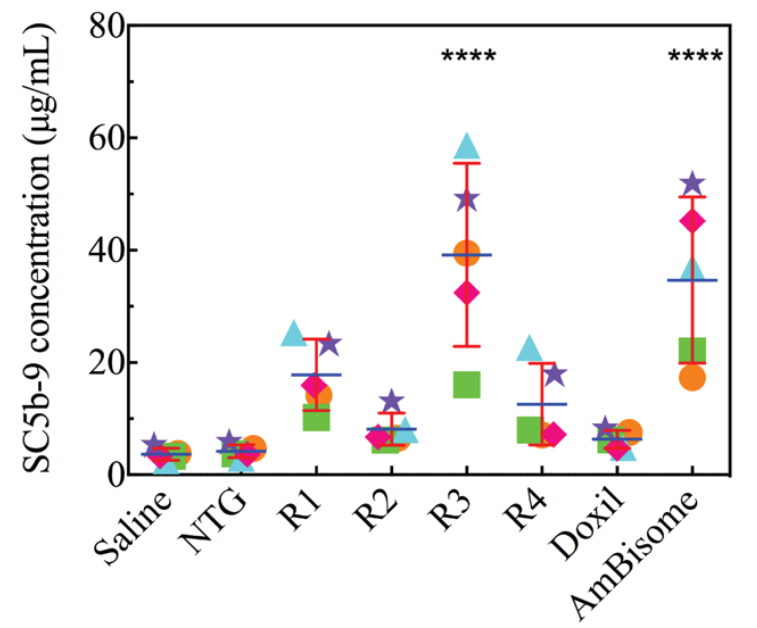

C

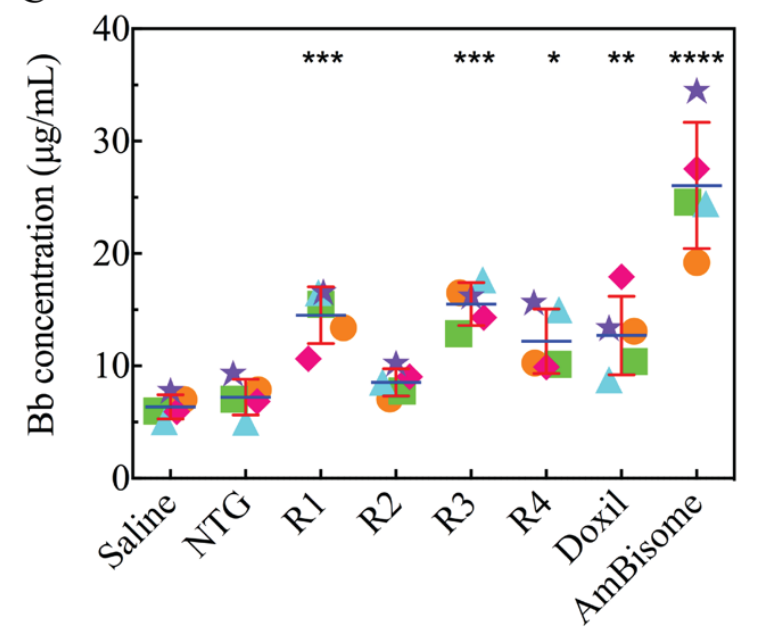

E

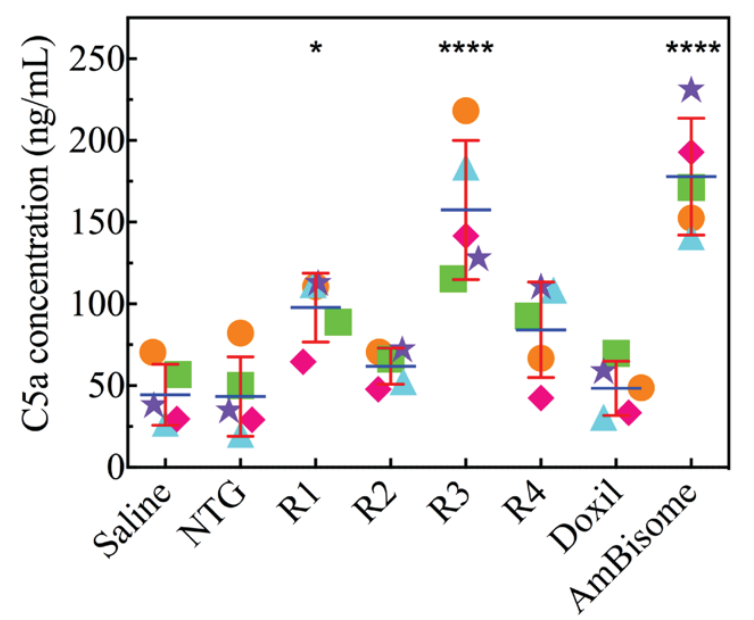

B

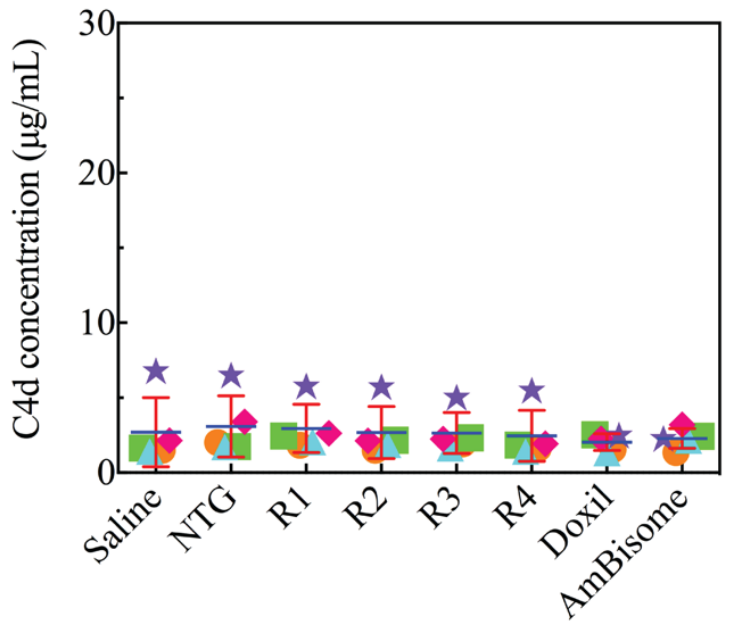

D

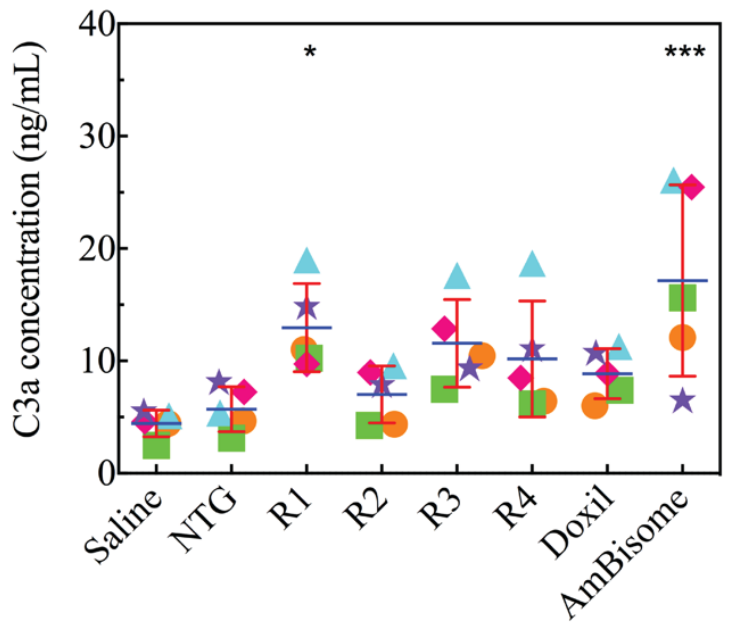

Donor \#1

Donor \#2

Donor \#3

Donor \#4

Donor \#6

Figure 7. The levels of SC5b-9 (A), C4d (B), Bb (C), C3a (D), and C5a (E) complement proteins. Human sera from five independent donors were incubated for a period of 40 minutes at a temperature of $37^{\circ} \mathrm{C}$ with saline, nitroglycerin (NTG), Rad-PC-Rad liposomal suspensions of selected composition (R1, R2, R3, R4), Doxil ${ }^{\circledR}$ and AmBisome ${ }^{\circledR}$. Saline solution was chosen as negative control. NTG was used as another negative control. Non-PEGylated liposomal formulations caused a higher level of $C$ activation, mainly via the alternative pathway. The activation of the $C$ cascade resulted in the increased production of C5a anaphylatoxin. The positive control zymosan caused substantially higher levels of complement activation. The data are represented as mean, including error bars derived from the standard deviation among the five donors. Each symbol and color represent data from a single donor. Significance of differences among the groups was determined by ordinary one-way ANOVA, followed by Dunnett's multiple comparisons test. P-values lower than 0.05 were considered as statistically significant. 


\section{Limitations of the study}

Several studies on adverse reactions, which were very recently published, are based on six or less donors [17, 31, 44]. Thus, the choice of six donors in the present study seems to be reasonable. One out of the six donors showed a specific behavior, which is similar to reactions of some individuals to liposomal drugs. Therefore, a substantially higher number of donors should be incorporated into future studies and the presented results have a preliminary character. The present results, however, could support the selection of the number of donors. In literature, one usually finds numbers of ten and above $[32,34,45]$.
We hypothesize that the false positive results, as observed in IL-8 production originate from the limitations of the multiplex array system, which necessitates the testing of the samples at multiple dilutions to detect lower and higher abundance cytokines. Another challenge in cytokine detection is their short half-live, such as six to seven minutes for TNF- a [46].

Furthermore, the prepared liposomes exhibited a wide variety of shapes and have to be regarded as a mixture of spherical and nonspherical species. Additional efforts have to be invested to obtain a homogeneous and uniform size and shape distribution.

\section{Conclusions}

The extensive research interest of nanomedicines, whether biologically derived or synthetically created, draws attention to their immunotoxicity. Nanometer-sized species interact with the immune system according to their morphology and composition. $\mathrm{C}$ activation and cytokine response can induce immune-stimulation and potentially life-threatening conditions, including anaphylaxis and cytokine storm. Therefore, we have carefully studied the interactions of the artificially synthesized Rad-PC-Rad liposomes with cellular and humoral components of the innate immune system in human blood and obtained promising results. In summary, the experimental results indicate that Rad-PC-Rad liposomes are promising shear-responsive nano-containers and related in vivo experiments could be foreseen in near future.

\section{Acknowledgments}

S.M. acknowledges the financial support of the Swiss Government via the Excellence Scholarship Program for Foreign Scholars and Artists (2015-2018 a.y.). A.Z and F.N. acknowledge funding by the Swiss National Centre of Competence in Research in Chemical Biology. T.M., G.T.K. and J.S. acknowledge the supports by the European Union Seventh Framework Program grants NMP-2012309820 (NanoAthero) and NMP-2013-602923 (TheraGlio) and the Applied Materials and Nanotechnology Center of Excellence at Miskolc University, Hungary. We thank PSI EM Facility for the cryo-TEM support.

\section{Conflict of interest}

The authors have no relevant financial or non-financial interests to disclose. For signed statements contact the journal office edidor@precisionnanomedicine.com.

Quote this article as Matviykiv S, Buscema M, Gerganova G, Mészáros T, Kozma GT, Mettal U, Neuhaus F, Ishikawa T, Szebeni J, Zumbuehl A, Müller B., Immunocompatibility of Rad-PC-Rad liposomes in vitro, based on human complement activation and cytokine release. Precis. Nanomed. 2018;1(1):43-62, https://doi.org/10.29016/180419.2

\section{References:}

1. Neuhaus, F., et al., Synthesis and biophysical characterization of an odd-numbered 1,3diamidophospholipid. $\quad$ Langmuir 2018, 34(10): p. 3215-3220, DOI: 10.1021/acs.langmuir.7b04227.

2. Farokhzad, O.C. and R. Langer, Impact of nanotechnology on drug delivery. ACS Nano, 2009, 3(1): p. 16-20, DOI: 10.1021/nn900002m.

3. Shi, J., et al., Nanotechnology in drug delivery and tissue engineering: from discovery to applications. Nano Letters, 2010, 10(9): p. 3223-3230, DOI: 10.1021/nl102184c. 
4. Holme, M.N., et al., Shear-stress sensitive lenticular vesicles for targeted drug delivery. Nature Nanotechnology, 2012, 7(8): p. 536-543, DOI: 10.1038/nnano.2012.84.

5. Saxer, T., A. Zumbuehl, and B. Müller, The use of shear stress for targeted drug delivery. Cardiovascular Research, 2013, 99(2): p. 328-333, DOI: 10.1093/cvr/cvt102.

6. Szebeni, J. and S.M. Moghimi, Liposome triggering of innate immune responses: a perspective on benefits and adverse reactions: biological recognition and interactions of liposomes. Journal of Liposome Research, 2009, 19(2): p. 85-90, DOI: 10.1080/08982100902792855.

7. Szebeni, J., et al., Liposome-induced complement activation and related cardiopulmonary distress in pigs: factors promoting reactogenicity of Doxil and AmBisome. Nanomedicine: Nanotechnology, Biology and Medicine, 2012, 8(2): p. 176-184, DOI: 10.1016/j.nano.2011.06.003.

8. Szebeni, J., Complement activation-related pseudoallergy: a stress reaction in blood triggered by nanomedicines and biologicals. Molecular Immunology, 2014, 61(2): p. 163-173, DOI: 10.1016/j.molimm.2014.06.038.

9. Nilsson, B., et al., The role of complement in biomaterial-induced inflammation. Molecular Immunology, 2007, 44(1): p. 82-94, DOI: 10.1016/j.molimm.2006.06.020.

10. Owen, J.A., J. Punt, and S.A. Stranford, Kuby immunology. 2013: WH Freeman New York, ISBN: 1464137846.

11. Matviykiv, S., et al., Liposomes: bio-inspired nano-containers for physically triggered targeted drug delivery. Proceedings of SPIE 10162, 2017, 10162: p. 101620A, DOI: $10.1117 / 12.2258378$.

12. Szebeni, J., et al., Complement activation-related cardiac anaphylaxis in pigs: role of C5a anaphylatoxin and adenosine in liposome-induced abnormalities in ECG and heart function. American Journal of Physiology-Heart and Circulatory Physiology, 2006, 290(3): p. H1050H1058, DOI: 10.1152/ajpheart.00622.2005.

13. Landesman-Milo, D. and D. Peer, Altering the immune response with lipid-based nanoparticles. Journal of Controlled Release, 2012, 161(2): p. 600-608, DOI: 10.1016/j.jconrel.2011.12.034.

14. Szebeni, J., Hemocompatibility testing for nanomedicines and biologicals: predictive assays for complement mediated infusion reactions. European Journal of Nanomedicine, 2012, 4(1): p. 33-53, DOI: 10.1515/ejnm-2012-0002.

15. Dobrovolskaia, M.A., Pre-clinical immunotoxicity studies of nanotechnology-formulated drugs: challenges, considerations and strategy. Journal of Controlled Release, 2015, 220: p. 571-583, DOI: $10.1016 /$ j.jconrel.2015.08.056.

16. European Medicine Agency. Reflection paper on the data requirements for intravenous products developed with reference to an innovator liposomal product. EMA/CHMP/806058/2009/Rev. 02, 2013,

17. Wolf-Grosse, S., et al., Iron oxide nanoparticles induce cytokine secretion in a complementdependent manner in a human whole blood model. International Journal of Nanomedicine, 2017, 12: p. 3927, DOI: 10.2147/IJN.S136453.

18. Buscema, M., et al., Immunological response to nitroglycerin-loaded shear-responsive liposomes in vitro and in vivo. Journal of Controlled Release, 2017, 264: p. 14-23, DOI: 10.1016/j.jconrel.2017.08.010.

19. Stalder, E. and A. Zumbuehl, Phosphate test 2.0. CHIMIA International Journal for Chemistry, 2013, 67(11): p. 819-821, DOI: 10.2533/chimia.2013.819.

20. Uskoković, V., Dynamic light scattering based microelectrophoresis: main prospects and limitations. Journal of Dispersion Science and Technology, 2012, 33(12): p. 1762-1786, DOI: 10.1080/01932691.2011.625523.

21. Silvander, M., Steric stabilization of liposomes - a review. Lipid and Polymer-Lipid Systems, 2002: p. 35-40, DOI: 10.1007/3-540-45291-5_5.

22. Garbuzenko, O., et al., Electrostatics of PEGylated micelles and liposomes containing charged and neutral lipopolymers. Langmuir, 2005, 21(6): p. 2560-2568, DOI: 10.1021/la0479105.

23. Liebi, M., et al., Magnetically enhanced bicelles delivering switchable anisotropy in optical gels. ACS Applied Materials \& Interfaces, 2014, 6(2): p. 1100-1105, DOI: 10.1021/am4046469. 
24. Kuntsche, J., J.C. Horst, and H. Bunjes, Cryogenic transmission electron microscopy (cryoTEM) for studying the morphology of colloidal drug delivery systems. International Journal of Pharmaceutics, 2011, 417(1): p. 120-137, DOI: 10.1016/j.ijpharm.2011.02.001.

25. Johnsson, M. and K. Edwards, Liposomes, disks, and spherical micelles: aggregate structure in mixtures of gel phase phosphatidylcholines and poly(ethylene glycol)-phospholipids. Biophysical Journal, 2003, 85(6): p. 3839-3847, DOI: 10.1016/S0006-3495(03)74798-5.

26. Almgren, M., K. Edwards, and G. Karlsson, Cryo transmission electron microscopy of liposomes and related structures. Colloids and Surfaces A: Physicochemical and Engineering Aspects, 2000, 174(1): p. 3-21, DOI: 10.1016/S0927-7757(00)00516-1.

27. Moghimi, S.M. and I. Hamad, Liposome-mediated triggering of complement cascade. Journal of Liposome Research, 2008, 18(3): p. 195-209, DOI: 10.1080/08982100802309552.

28. Moghimi, S.M., et al., Material properties in complement activation. Advanced Drug Delivery Reviews, 2011, 63(12): p. 1000-1007, DOI: 10.1016/j.addr.2011.06.002.

29. Szebeni, J. and Y. Barenholz, Complement activation, immunogenicity, and immune suppression as potential side effects of liposomes. Handbook of Harnessing Biomaterials in Nanomedicine: Preparation, Toxicity, and Applications, Pan Stanford Publishing Pte. Ltd., Singapore, 2012: p. 309-334, DOI: 10.4032/9789814364270.

30. Devine, D.V., et al., Liposome - complement interactions in rat serum: implications for liposome survival studies. Biochimica et Biophysica Acta, 1994, 1191(1): p. 43-51, DOI: 10.1016/0005-2736(94)90231-3.

31. Wibroe, P.P., et al., Bypassing adverse injection reactions to nanoparticles through shape modification and attachment to erythrocytes. Nature Nanotechnology, 2017, 12(6): p. 589-594, DOI: 10.1038/nnano.2017.47.

32. Van Den Hoven, J.M., et al., Complement activation by PEGylated liposomes containing prednisolone. European Journal of Pharmaceutical Sciences, 2013, 49(2): p. 265-271, DOI: 10.1016/j.ejps.2013.03.007.

33. Chanan-Khan A J, S.J., Savay S, Liebes L, Rafique N M, Alving C R, Muggia F M., Complement activation following first exposure to pegylated liposomal doxorubicin (Doxil囚): possible role in hypersensitivity reactions. Annals of Oncology, 2003, 14(9): p. 1430-1437, DOI: $10.1093 /$ annonc/mdg374.

34. Szebeni, J., et al., Role of complement activation in hypersensitivity reactions to doxil and hynic PEG liposomes: experimental and clinical studies. Journal of Liposome Research, 2002, 12(12): p. 165-172, DOI: 10.1081/LPR-120004790.

35. Moghimi, S.M., et al., Methylation of the phosphate oxygen moiety of phospholipid-methoxy (polyethylene glycol) conjugate prevents PEGylated liposome-mediated complement activation and anaphylatoxin production. The FASEB Journal, 2006, 20(14): p. 2591-2593, DOI: 10.1096/fj.06-6186fje.

36. Szebeni, J., et al., Activation of complement by therapeutic liposomes and other lipid excipientbased therapeutic products: prediction and prevention. Advanced Drug Delivery Reviews, 2011, 63(12): p. 1020-1030, DOI: 10.1016/j.addr.2011.06.017.

37. Szebeni, J., et al., The role of complement activation in hypersensitivity to pegylated liposomal doxorubicin (Doxil $\mathbb{R})$. Journal of Liposome Research, 2000, 10(4): p. 467-481, DOI: 10.3109/08982100009031112.

38. COMPATH. Serum preparation information sheet. Available from: http://compath.com.au/user assets/17dbd37927d046b9835ed2a829f9709e1c22f7d9/serum pr eparation may2015.pdf.

39. Bugna, S., et al., Surprising lack of liposome-induced complement activation by artificial 1, 3diamidophospholipids in vitro. Nanomedicine: Nanotechnology, Biology and Medicine, 2016, 12(3): p. 845-849, DOI: 10.1016/j.nano.2015.12.364.

40. Andersen, A.J., et al., Complement: alive and kicking nanomedicines. Journal of Biomedical Nanotechnology, 2009, 5(4): p. 364-372, DOI: 10.1166/jbn.2009.1045.

41. Zhou, X., et al., Conceptual and methodological issues relevant to cytokine and inflammatory marker measurements in clinical research. Current Opinion in Clinical Nutrition and Metabolic Care, 2010, 13(5): p. 541, DOI: 10.1097/MCO.0b013e32833cf3bc. 
42. Jones, C.F. and D.W. Grainger, In vitro assessments of nanomaterial toxicity. Advanced Drug Delivery reviews, 2009, 61(6): p. 438-456, DOI: 10.1016/j.addr.2009.03.005.

43. Ilinskaya, A.N., et al., Induction of oxidative stress by Taxol ${ }^{\circledR}$ vehicle Cremophor-EL triggers production of interleukin-8 by peripheral blood mononuclear cells through the mechanism not requiring de novo synthesis of $m R N A$. Nanomedicine: Nanotechnology, Biology and Medicine, 2015, 11(8): p. 1925-1938, DOI: 10.1016/j.nano.2015.07.012.

44. Wibroe, P.P., et al., An integrated assessment of morphology, size, and complement activation of the PEGylated liposomal doxorubicin products Doxil ${ }^{\circledR}$, Caelyx ${ }^{\circledR}$, DOXOrubicin, and SinaDoxosome. Journal of Controlled Release, 2016, 221: p. 1-8, DOI: 10.1016/j.jconrel.2015.11.021.

45. Benasutti, H., et al., Variability of Complement Response toward Preclinical and Clinical Nanocarriers in the General Population. Bioconjugate chemistry, 2017, 28(11): p. 2747-2755, DOI: 10.1021/acs.bioconjchem.7b00496.

46. Beutler, B.A., I.W. Milsark, and A. Cerami, Cachectin/tumor necrosis factor: production, distribution, and metabolic fate in vivo. The Journal of Immunology, 1985, 135(6): p. 39723977, OSTI: 5529162. 